\title{
Prevalencia de violencia doméstica en la ciudad de Durango*
}

Gloria Alvarado-Zaldívar, M. en C., J1) Jaime Salvador-Moysén, M.I.S.P., ${ }^{(1)}$

Sergio Estrada-Martínez, Lic. en Ing., ${ }^{(1)}$ Alberto Terrones-González, Lic. en Psic. ${ }^{(2)}$

\section{A lvarado-Zaldívar G, Salvador-Moysén J, Estrada-Martínez S, Terrones-González A, Prevalencia de violencia doméstica en la ciudad de Durango. Salud Publica Mex 1998;40:481-486.}

\begin{abstract}
Resumen
Objetivo. C aracterizar y determinar, desde un enfoque de género, la prevalencia de los diferentes tipos de violencia que se presentan en la ciudad de D urango, D go., México. Material y métodos. C on un diseño transversal se entrevistaron 384 mujeres casadas, 0 bien, unidas al mo mento del estudio 0 alguna vez, residentes de la ciudad de D urango. El tamaño de la muestra se distribuyó en forma proporcional en seis sectores de la ciudad, seleccionados al azar y representativos de los niveles socioeconómicos alto, medio y bajo. En cada sector se realizaron 64 entrevistas. El instrumento de medición fue un cuestionario con 184 preguntas cerradas y 22 abiertas, que incluía datos de identificación, socio demográficos y reproductivos, así co mo reactivos específicos para la violencia física, emocional y sexual. Resultados. La mediana de edad del grupo estudiado fue de 41.5 años, con un rango de 12 a 48 años. La prevalencia de violencia doméstica fue: alguna forma de violencia sexual, 42\%; física, 40\%, y emo cional, $39 \%$. Conclusiones. Se reconoce que el problema de la violencia, en sus diferentes formas, es un hecho altamente prevalente que pone en peligro el bienestar del núcleo familiar. Se observó una mayor prevalencia de violencia doméstica en presencia de factores tales como: antecedente de violencia, alcoholismo y/o consumo de drogas en algún miembro de la familia.
\end{abstract}

Palabras clave: violencia doméstica/prevalencia; género; México

\author{
Alvarado-Zaldívar G, Salvador-Moysén J, \\ Estrada-Martínez S, Terrones-González A, \\ Prevalence of domestic violence \\ in the city of Durango, Mexico. \\ Salud Publica Mex 1998;40:481-486.
}

\begin{abstract}
A bstract
Objective. To characterize and determine the prevalence of the different types of gender-associated violence in the city of Durango, Mexico. Material and methods. W ith a transversal design, 384 women residents of the city of Durango, either living with or having lived with someone before, were interviewed. The sample was proportionately distributed in 6 city sectors which were randomly chosen and representative of the high middle and low socioeconomic levels. Sixty-four inter views were conducted in each city sector. The questionnaire consisted of 184 closed and 22 open questions, including identification, socio demographic and reproductive data, as well as specific questions on physical, emotional and sexual violence. Results. Median age of the studied group was 41.5 years, ranging from 12 to 48 years.Prevalence of domestic violence was: sexual $42 \%$; physical $40 \%$; emotional $39 \%$. Conclusions. The problem of violence, in its different forms, is a highly prevalent factor wich jeopardizes the welfare of the family nucleus. Higher prevalence values of domestic violence were detected in the presence of factors such as violence antecedents, alcoholism and/or drug consumption by some member of the family.
\end{abstract}

Key words: domestic violence/prevalence; gender; Mexico

\footnotetext{
* Este trabajo se realizó gracias al apoyo brindado por el Consejo $\mathrm{N}$ acional de Ciencia yTecnología, con el financiamiento al proyecto 1154P-M.
}

(1) Instituto de Investigación Científica, U niversidad Juárez del Estado de Durango (UJED ), México.

(2) Dirección de 0 rientación Educativa, UJED, México.

Fecha de recibido: 9 de octubre de 1997 - Fecha de aprobado: 26 de agosto de 1998 
a violencia y el maltrato en el ámbito familiar se reconocieron como un problema social únicamente hasta 1960, cuando algunos autores describieron el síndrome del niño maltratado. ${ }^{1}$ Antes de esa fecha, la violencia contra el género femenino era considerada como un fenómeno poco frecuente, estaba catalogado como anormal y se le atribuía a personas con trastornos psicopatológicos.

En los últimos 20 años, los resultados de diversas investigaciones han demostrado que la violencia doméstica no es $\tan$ rara, que es una violencia de género y que, en la mayoría de los casos, la ejerce el compañero íntimo o el excompañero de la mujer.

La conducta violenta, entendida como el uso de la fuerza para la resolución de conflictos personales, surge cuando existe un desequilibrio de poder, permanente o momentáneo, en el cual se establece una relación de abuso. Por medio de ese tipo de conducta, se busca someter, doblegar o subordinar al otro miembro de la relación, ocasionándole daño físico, psíquico y / o económico. ${ }^{2}$

No existe una definición universal de violencia; $\sin$ embargo, en 1996 un grupo de expertos, convocado por la Organización Mundial de la Salud, decidió adoptar la definición que utilizó en 1993 la Asamblea General de las Naciones Unidas, misma que señala que la violencia contra la mujer es "cualquier acto de violencia basado en el género, que resulta o puede resultar en daño o sufrimiento físico, mental o sexual en la mujer, incluyendo la amenaza de dichos actos, la coerción o la privación arbitraria de la libertad, tanto en la vida pública como en la privada" ${ }^{3,4}$ Por otra parte, por violencia familiar se entienden las distintas formas de relación abusiva que caracterizan, de modo permanente o cíclico, al vínculo familiar.

Las causas de este fenómeno son múltiples; se han señalado factores tales como problemas de personalidad y de dinámica interpersonal familiar, situaciones variables como el nivel de pobreza y el estrés económico, así como normas culturales que dan soporte a la violencia de género o a la provocada por la desigualdad social. ${ }^{5}$

Se estima que en el mundo más de 5000000 de mujeres al año son víctimas de abuso físico severo por parte de sus esposos, y que de 50 a $60 \%$ son violadas por ellos mismos. ${ }^{6}$

Se ha señalado que $75 \%$ de los casos de violencia intrafamiliar corresponden a maltrato hacia la mujer, $2 \%$ a abuso hacia el hombre, y $23 \%$, a casos de violencia cruzada. $^{2}$

Desde la perspectiva epidemiológica, es cada vez más evidente que la violencia doméstica se presenta en diferentes formas, y que recae de distintas maneras sobre una variedad de grupos, como los constituidos por mujeres, niños y adolescentes. ${ }^{3}$
En la encuesta que la Asociación Contra la Violencia hacia la Mujer, A.C. (COVAC) llevó a cabo en 1995, y que incluyó nueve ciudades de la República mexicana, se encontró que la prevalencia de violencia hacia la mujer fue: física, con $81 \%$; emocional o psicológica, con $76 \%$, y sexual, con $32 \%$. Las conductas más comunes para ocasionar daño fueron: propinar golpes, gritar y proferir groserías, así como la práctica de la violación. En la mayoría de los casos el agresor fue el hombre. ${ }^{7} \mathrm{El}$ Banco Mundial estima que a causa de la violencia doméstica las mujeres en edad reproductiva pierden entre 5 y $16 \%$ de años de vida saludable, es decir, uno de cada cinco días de salud. ${ }^{8}$

Debido a que la violencia en un micronivel como es el doméstico ha sido reconocida como un problema de salud pública y a que existe poca información epidemiológica al respecto en México, el objetivo de este estudio ha sido caracterizar, desde un enfoque de género, los diferentes tipos de violencia que se presentan en la ciudad de Durango, Dgo., México.

\section{Material y métodos}

Mediante un diseño transversal, de junio a diciembre de 1996 se entrevistaron 384 mujeres casadas, unidas al momento del estudio o alguna vez, residentes de la ciudad de Durango, Dgo. El tamaño de la muestra se estimó mediante la siguiente fórmula:

$$
\mathrm{n}=\mathrm{p}(1-\mathrm{p})(\mathrm{z} \text { alfa })^{2} / \mathrm{d}^{2}
$$

donde $\mathrm{z}$ alfa $=1.96 \mathrm{y} \mathrm{d}=.05$. El universo muestral $(\mathrm{n}=384)$ se distribuyó en seis colonias o fraccionamientos seleccionados al azar, representativos de los niveles socioeconómicos alto, medio y bajo. Dichas categorías se tomaron del Periódico Oficial del Gobierno Constitucional del Estado de Durango de 1991, actualmente vigente. ${ }^{9}$

Se elaboró un cuestionario semiestructurado con 184 preguntas cerradas y 22 abiertas, integrado por la ficha de identificación de la entrevistada y los capítulos para evaluar las dimensiones sociodemográfica y reproductiva, así como por reactivos específicos para violencia emocional o psicológica, física y sexual, con el propósito de detectar algún tipo de violencia doméstica. Para la construcción de cada reactivo, se hizo la correspondiente revisión en la literatura ${ }^{7-10}$ y se contó con la asesoría de un experto en la materia. Dos revisores analizaron el documento de manera independiente, a fin de obtener un consenso alrededor de la versión final del instrumento (validez de contenido).

En cada sector se llevaron a cabo 64 entrevistas, seleccionando al azar los domicilios. Personal de enfermería, trabajo social y psicología, de ambos sexos y con 
edades de entre 20 y 35 años, previamente capacitado para el efecto, se encargó de recolectar la información La supervisión del trabajo de campo estuvo a cargo de una antropóloga social. Con el objeto de facilitar las respuestas, las entrevistas fueron confidenciales y anónimas.

Antes de aplicar este instrumento se efectuó una prueba piloto de 30 encuestas para medir su confiabilidad interna; no se incluyeron nuevas variables y sólo se modificó la construcción de algunos reactivos. Los datos se capturaron y procesaron con el paquete computacional epidemiológico Epi Info 6.0.

\section{A nálisis estadístico}

Se aplicaron procedimientos estadísticos descriptivos para el cálculo de la media, la mediana y el rango, así como inferenciales para la estimación de razones de prevalencia y sus intervalos de confianza al 95\%; para tal efecto se conformaron dos grupos: uno de casos y otro de no casos. Para operacionalizar el análisis estadístico se estableció un punto de corte en cuanto al número de reactivos positivos en cada una de las dimensiones. Para la esfera emocional se seleccionaron 15 reactivos del instrumento, considerados como los más significativos, y se estableció el punto de corte en tres reactivos positivos. Para la violencia física, de 10 reactivos seleccionados, dos positivos se utilizaron para fijar el punto de corte, y para la dimensión sexual, donde se incluyeron siete reactivos, el límite se estableció en dos respuestas positivas. Se construyó un modelo de análisis de regresión logística no condicionada ${ }^{11}$ para cada una de las modalidades de violencia explorada (emocional, física y sexual). Se obtuvo el alfa de Cronbach ${ }^{12}$ para medir la confiabilidad del instrumento en cada dimensión explorada.

\section{Resultados}

La edad del grupo estudiado presentó una mediana de 41.5 años, con un rango de 12 a 48 años. En el cuadro I se muestran algunas de las características sociodemográficas y reproductivas de dicho grupo. En relación con el estado civil, predominaron las mujeres casadas (83\%).

En cuanto a la escolaridad, $65 \%$ de las entrevistadas refirieron tener estudios de secundaria o niveles de enseñanza más altos, y $35 \%$ se ubicó en el nivel de primaria. La mediana de escolaridad fue de nueve años, con un rango de 1 a 23 años. Respecto a la ocupación, la mayoría $(90 \%)$ se ubicó en la categoría de ama de casa.

En relación con las características reproductivas, el inicio de vida sexual activa presentó una mediana de 20 años, con un rango de 12 a 34 años. La edad al primer embarazo tuvo una mediana de 21 años, con un rango de 13 a 34 años. La mediana para el total de embarazos fue de cuatro con un rango de 1 a 9.

salud pública de méxico / vol.40, no.6, noviembre-diciembre de 1998

\section{Cuadro I \\ Caracteristicas Sociodemográficas y reproductivas de mujeres objeto de Violencia. Durango, MéxıCO,1996.}

\begin{tabular}{lrc} 
Variable & $\mathrm{n}$ & $\%$ \\
$\begin{array}{lr}\text { Estado civil } \\
\text { Casada }\end{array}$ & 320 & 83 \\
\hline Unida actualmente 0 alguna vez & 64 & 17
\end{tabular}

Escolaridad

\begin{tabular}{lll} 
Primaria incompleta & 58 & 15 \\
\hline Primaria completa & 77 & 20 \\
\hline Secundaria & 77 & 20 \\
\hline Preparatoria & 93 & 24 \\
\hline Profesionista & 79 & 21
\end{tabular}

0 cupación

\begin{tabular}{lrr} 
A ma de casa & 344 & 90 \\
\hline Comerciante 0 ayudante doméstica & 23 & 6 \\
\hline
\end{tabular}

Empleada y profesionista

Ingreso mensual (salarios mínimos)

\begin{tabular}{lrc} 
N inguno & 304 & 79 \\
\hline 1.1 a 2 & 26 & 7 \\
\hline 2.1 a 5 & 21 & 5 \\
\hline 5.1 y más & 25 & 7 \\
\hline No contestó & 8 & 2
\end{tabular}

$\mathrm{N}=384$

Fuente: encuesta directa

La prevalencia de alguna forma de violencia emocional fue de 39\%, y su expresión varió de enojo del compañero (muy frecuente), a otras modalidades tales como: no le permite trabajar, no le dice cuánto gana, la amenaza con gestos y actitudes (situaciones que se presentaron con menos frecuencia).

La prevalencia de violencia física fue de $40 \%$, y sus diferentes formas de expresión se describen en el cuadro II. Cabe señalar que cuando a las entrevistadas se les preguntó si alguna vez su esposo o compañero las había golpeado, $80 \%$ dijo que no, y cuando se les preguntó si les había hecho alguna "cosa", $40 \%$ contestó afirmativamente.

Las principales razones por las cuales la mujer es golpeada son: en primer lugar por chismes, porque "no lo cela", por salirse de la casa sin avisar, por mostrarse celosa, porque él está de mal humor, porque ella defiende a los hijos y por ser mala ama de casa. En segundo lugar, porque él está celoso; en tercer lugar, porque él había ingerido alcohol cuando la golpeó, y en cuarto lugar, fue sin motivo. En esta sección sólo contestaron 70 entrevistadas.

Las principales consecuencias de los golpes fueron, en orden de frecuencia: heridas en la boca, sangrado por nariz, ruptura de tímpano e inflamación en tórax. 


\section{Cuadro II \\ DistribuCIÓN PORCENTUAL DE ALGUNAS FORMAS DE VIOLENCIA EMOCIONAL, FISICA Y SEXUAL EJERCIDA sobre las mujeres. Durango, México, 1996}

\begin{tabular}{lcc}
\multicolumn{1}{c}{ Tipo de violencia } & $n$ & $\%$ \\
& & \\
Violencia emocional* & & \\
Se enoja con frecuencia & 274 & 72.1 \\
\hline N o le permite trabajar & 146 & 38.2 \\
\hline N o sabe cuánto gana & 144 & 37.9 \\
\hline La ha culpado de lo que sucede en la familia & 114 & 29.9 \\
\hline Le ha dicho que está loca & 97 & 25.5 \\
\hline La ha amenazado con gestos o acciones & 96 & 25.2 \\
\hline La ha amenazado con herirla o matarla & 64 & 16.8 \\
\hline La ha amenazado con abandonarla & 62 & 16.3 \\
\hline N o comparte con usted la toma de decisiones & 55 & 14.4 \\
\hline N o le permite tener amistades & 44 & 11.5 \\
\hline Le cuenta de sus relaciones con otras personas & 30 & 10.0 \\
\hline La ha obligado a pedir dinero & 23 & 6.0 \\
\hline La ha amenazado con suicidarse & 23 & 6.0 \\
\hline Le ha escupido & 10 & 2.6 \\
\hline La ha obligado a cometer algún delito & 5 & 1.3 \\
& & \\
Violencia física* & & \\
La ha aventado & 106 & 27.8 \\
\hline Le ha dado cachetadas & 81 & 21.3 \\
\hline La ha estrellado contra la pared & 36 & 9.4 \\
\hline Le ha dado patadas & 33 & 8.7 \\
\hline Ha usado contra usted algún objeto & 21 & 5.5 \\
\hline La ha mordido & 13 & 3.4 \\
\hline Ha usado contra usted un arma de fuego & 12 & 3.6 \\
\hline Ha usado contra usted un arma blanca & 10 & 2.6 \\
\hline La ha cortado & 8 & 2.1 \\
\hline La ha quemado & 4 & 1.1
\end{tabular}

Violencia sexual*

La ha obligado a tener relaciones sexuales cuando usted no quiere

La ha go lpeado durante la relación sexual

Critica su funcionamiento sexual

La ha obligado a tener relaciones sexuales

de alguna forma que a usted no le guste

La ha obligado a tener relaciones sexuales

cuando está usted enferma

La ha obligado a tener relaciones sexuales

con otras personas

La ha obligado a tener relaciones sexuales

delante de sus hijos

$64 \quad 16.8$

$61 \quad 16.0$

$42 \quad 11.1$

7.6

$29 \quad 7.6$

$18 \quad 4.7$

10 2.6

$\mathrm{N}=384$

* Se refiere a la relación que existe entre la entrevistada y su esposo 0 compañero

‡ Se consideró que este acto produce más daño emocional que físico

Fuente: encuesta directa

De las entrevistadas, $13 \%$ recibieron golpes durante el embarazo; de ellas, $44 \%$ presentó amenaza de aborto; $12 \%$, aborto, y $2 \%$, muerte fetal. La violencia física apa- reció cuando las víctimas ya estaban casadas o unidas, en $96 \%$ de los casos durante el primer año de unión.

De las mujeres golpeadas, $35 \%$ acudió a sus padres o familiares; $14 \%$, con la policía judicial; $14 \%$, con amigas; $10 \%$ buscó la ayuda de algún psicólogo; $7 \%$, de los suegros; $7 \%$, de los hijos; $7 \%$, del médico, y $6.5 \%$ recurrió al Sistema Nacional para el Desarrollo Integral de la Familia-DIF.

Del total de entrevistadas, 15\% declaró haber golpeado al esposo por las siguientes razones: por coraje, en defensa propia, porque es alcohólico, por desesperación y por maltrato a los hijos.

La prevalencia de alguna forma de violencia sexual fue de $42 \%$; se observó que las relaciones sexuales sin el consentimiento de la mujer constituyen la agresión más frecuente en esta dimensión (cuadro II).

Otras formas de violencia sexual referidas por las integrantes del grupo fueron: intento de violación por parte de personas distintas al compañero o esposo $(6 \%)$. Diecinueve de las entrevistadas declararon haber sido violadas, pero sólo cuatro $(21 \%)$ contestaron quién había sido el agresor: en un caso fue el padre, y en los otros fueron la madre, un tío y el suegro de una prima. El resultado de la violación se tradujo en un embarazo a término en uno de los casos, en lesiones físicas en otro y en crisis nerviosa y depresión en los otros dos.

Los resultados del alfa de Cronbach -indicativos de la confiabilidad del instrumento utilizado- fueron los siguientes: violencia emocional, 0.839 ; física, 0.805 , y sexual, 0.656 .

En el cuadro III se muestra la razón de prevalencia, ajustada por el nivel socioeconómico y con un intervalo de confianza al 95\%, de los indicadores de las distintas variables que se identificaron más frecuentemente ante la presencia de algún tipo de violencia. Se aprecia que los indicadores observados en relación con la violencia emocional y física son semejantes, a excepción del número de hijos y de la agresión física a los hermanos por parte del padre. Para la violencia sexual, el antecedente de violencia física, así como los problemas de alcoholismo y / o drogadicción en algún miembro de la familia, fueron las variables más consistentes. El nivel socioeconómico no muestra indicios de ser una variable confusora.

Se estimaron las siguientes ecuaciones de regresión logística no condicional para cada uno de los tipos de violencia:

- La probabilidad de sufrir violencia en la esfera emocional $=-9.48+0.93$ estado civil (unida) +0.73 percibe salario +1.26 presente cuando el padre golpeaba a la madre +0.45 problemas de alcoholismo en la familia + 1.08 problemas de drogadicción en la fa- 


\section{Cuadro III \\ FACTORES PRESENTES EN LOS DifERENTES TIPOS DE VIOLENCIA, AJUSTADOS POR NIVEL SOCIOECONÓMICO. Durango, México, 1996.}

\begin{tabular}{|c|c|c|c|c|c|c|c|c|c|c|c|c|}
\hline & & & & & & & Tipo & de vio & Iencia & & & \\
\hline & & & & & Emocion & & & Física & & & Sexual & \\
\hline Variable & Indicador & & estos & $\mathrm{RP}$ & $\mathrm{RP}$ & IC (95\%) & $\mathrm{RP}$ & $\mathrm{RP}$ & IC $(95 \%)$ & $\mathrm{RP}$ & $\mathrm{RP}$ & IC $(95 \%)$ \\
\hline & & $\%$ & $\bar{n}$ & cruda & ajustada & & & ajustada & & & ajustada & \\
\hline Estado civil & Unida & 16.7 & 64 & 2.43 & 2.12 & $1.3-4.5$ & 2.14 & 2.0 & $1.0-4.5$ & & & \\
\hline Escolaridad & $<6$ años & 20.1 & 77 & 2.35 & 1.85 & $1.3-4.1$ & 2.08 & 1.87 & $1.0-4.3$ & & & \\
\hline Escolaridad del compañero & $<6$ años & 12.8 & 49 & 1.74 & 1.35 & $1.1-2.9$ & 2.2 & 2.09 & $1.0-4.4$ & & & \\
\hline Edad de la primera relación sexual & $<19$ años & 27.1 & 104 & 1.79 & 1.52 & $1.0-3.2$ & 2.7 & 2.68 & $1.3-5.3$ & & & \\
\hline Edad del primer embarazo & $<17$ años & 19.0 & 73 & 1.83 & 1.49 & $1.0-3.2$ & 4.9 & 5.30 & $2.4-9.9$ & & & \\
\hline N úmero de hijos & $\geq 6$ & 19.0 & 73 & 1.78 & 1.60 & & 2.2 & 2.21 & $1.0-4.7$ & & & \\
\hline Algún hijo aporta dinero & Sí & 29.8 & 115 & 2.07 & 1.93 & $1.2-3.3$ & & & & & & \\
\hline Escolaridad del padre & $=0$ & 27.9 & 107 & 1.55 & 1.39 & $1.0-2.5$ & & & & & & \\
\hline El padre le pegó & $=0$ & 31.5 & 121 & 1.91 & 1.88 & $1.2-3.0$ & 2.1 & 2.05 & $1.0-4.1$ & & & \\
\hline El padre le pegó a sus hermanos & Sí & 39.3 & 151 & & & & 2.2 & 2.24 & $1.1-4.6$ & & & \\
\hline La madre le pegó a sus hermanos & Sí & 56.7 & 218 & 1.53 & 1.49 & $1.0-2.3$ & & & & & & \\
\hline Alguien más le pegó & Sí & 18.9 & 73 & 1.89 & 1.89 & $1.0-3.3$ & 2.1 & 2.12 & $1.0-4.6$ & & & \\
\hline El padre go lpea a la madre & Sí & 22.8 & 88 & 1.74 & 1.47 & $1.0-2.9$ & 4.0 & 3.60 & $2.0-8.0$ & 2.5 & 2.45 & $1.2-5.0$ \\
\hline Estuvo presente cuando el padre & & & & & & & & & & & & \\
\hline golpeaba a su madre & Sí & 63.6 & 244 & 3.54 & 3.54 & $1.3-9.0$ & 1.85 & 1.91 & $1.0-4.0$ & & & \\
\hline Algún miembro de la familia & & & & & & & & & & & & \\
\hline con problemas de alcohol & Sí & 57.1 & 219 & 2.11 & 1.88 & $1.3-3.2$ & 3.6 & 3.5 & $1.6-8.4$ & 5.3 & 6.03 & $2.2-13.5$ \\
\hline Algún miembro de la familia & & & & & & & & & & & & \\
\hline con problemas de drogas & Sí & 12.5 & 48 & 2.94 & 2.76 & $1.4-6.1$ & 3.7 & 3.71 & $1.7-8.2$ & 3.0 & 2.93 & $1.3-6.7$ \\
\hline
\end{tabular}

Fuente: encuesta directa

milia; el estimador de máxima verosimilitud (Loglikelihood) fue de 230.93, y el valor de $p$, de $<0.001$.

- La probabilidad de sufrir violencia física=-8.19+ 0.61 estado civil (unida) + 0.15 edad al primer embarazo +0.93 el padre golpeaba a la madre +0.97 problemas de alcoholismo en la familia; el estimador de máxima verosimilitud fue de 201.14, y el valor de $p$, de $<0.001$.

- La probabilidad de sufrir violencia sexual $=-3.33+0.63$ estado civil (unida) +0.63 escolaridad del compañero +0.61 el padre golpeaba a la madre +0.83 problemas de drogadicción en la familia; el estimador de máxima verosimilitud fue de 431.37, y el valor de $p$, de $<0.05$

\section{Discusión}

De acuerdo con los datos que se han publicado, la violencia emocional o psicológica predomina sobre la violencia física y la sexual. ${ }^{7,10,13}$ Los resultados de este estudio difieren de esos hallazgos, ya que la forma de violencia más frecuente fue la sexual, seguida de la física y, por último, de la emocional o psicológica.

De las entrevistadas, $42 \%$ ha sido víctima de alguna forma de violencia sexual: las relaciones sexuales sin el consentimiento de la mujer y los golpes durante el coito fueron las agresiones más frecuentes en esta dimensión. Por otra parte, $40 \%$ de las participantes en este estudio son víctimas de la violencia física que ejerce el esposo, o bien, el compañero; $29 \%$ de ellas corren un elevado riesgo de morir por esa causa, pues han tratado de ahorcarlas y / o han usado contra ellas arma blanca, de fuego u objetos pesados.

Respecto a la frecuencia de violencia física durante el embarazo, los resultados son mayores a los observados por Valdez-Santiago, ${ }^{14}$ en relación con la violencia emocional, la forma más frecuente fue la amenaza y la intimidación, resultado que coincide con lo observado por la COVAC. ${ }^{7}$ 
Los factores socioculturales se encuentran entre los de mayor peso en relación con la violencia doméstica, razón por la cual muchas situaciones de inequidad y desigualdad no son percibidas como tales. Así, por ejemplo, el control económico y social (a estas mujeres no se les permite trabajar, tener amistades, o bien, su pareja les oculta la cantidad de salario que percibe) se presentó en $96 \%$ del grupo; sin embargo, eso es declarado como algo normal. Estos resultados difieren de los que encontraron Ramírez y Patiño. ${ }^{10}$

Respecto a la confiabilidad del instrumento, los resultados muestran que la exploración de la violencia emocional y física fue la más adecuada; la violencia sexual es un aspecto que requiere de una investigación más profunda.

La mayor prevalencia de violencia emocional se observó en mujeres con las siguientes características: unidas (estado civil), con una escolaridad menor a seis años, antecedentes de violencia física en la madre de la entrevistada, así como alcoholismo y / o drogadicción en algún miembro de la familia.

En relación con la violencia física, a los factores señalados con anterioridad se añaden la escolaridad del compañero, la edad de la primera relación sexual y la edad en el primer embarazo. Por último, se observó una mayor prevalencia de violencia sexual cuando hubo consumo de alcohol y/o drogas.

\section{Conclusiones}

- La prevalencia de violencia intrafamiliar más frecuente en las mujeres de la ciudad de Durango fue la sexual, seguida por la física y la emocional.

- En la mayoría de los casos la violencia física va acompañada de violencia emocional en forma de intimidación y abuso verbal.

- El antecedente de violencia en la entrevistada, así como el alcoholismo y/o consumo de drogas en algún miembro de la familia, son factores que se observaron consistentemente en las tres dimensiones exploradas.

- Sigue siendo difícil abordar el tema de la violación sexual, debido a que se considera como un asunto vergonzoso.

Si bien el diseño de este estudio es transversal y no se puede hablar de causalidad en términos metodológicos, sus resultados pueden ser útiles para el diseño de programas preventivos de violencia intrafamiliar.

Se considera que es necesario seguir adelante con la investigación en materia de violencia, en particular desde la perspectiva de género, la percepción que de ella tienen los diferentes miembros de una familia y las consecuencias que este fenómeno tiene sobre la salud.

\section{Agradecimientos}

Se agradece la valiosa asesoría de la doctora Luciana Ramos Lira, así como la colaboración de los alumnos de Psicología de la Universidad Autónoma de Durango en la fase de campo, y a la Licenciada en Enfermería Eva Ortíz, la revisión de las encuestas.

\section{Referencias}

1. Herrada-Huidobro A, N azar-Beutelspacher A, C assaball-N úñez M,VegaRamos R, N ava-Cruz CB. El niño maltratado en T laxcala: estudio de casos. Salud Publica Mex 1992;34(6):626-634.

2. C orsi J.Violencia intrafamiliar. U na mirada interdisciplinaria so bre un grave problema social. Buenos Aires: Paidós, 1994:1-160.

3. Health communications and public relations. Ginebra:W orld $\mathrm{H}$ ealth $\mathrm{O} \mathrm{r}$ ganization, 1996.

4. United $N$ ations. Report of theW orking $G$ roup onViolence A gainstW omen.Vienna. E/CN .6/W G.2/1992/1.3.

5.Stark E, Flitcraft A.W omen at risk:D omestic violence and women's health. California: Sage Publications, 1996:25.

6. Mehta P, Dandrea L. The battered woman. American Family Physician 1988:37:193-199.

7. D uarte P. Encuesta de opinión pública sobre la incidencia de violencia en la familia. A sociación Mexicana contra laViolencia hacia las Mujeres,A.C. (COVAC).México, D.F.:Fondo de Población de las $\mathrm{N}$ aciones Unidas/Procuraduría General de Justicia del D istrito Federal, 1995:17.

8. Heise L.Violencia contra la mujer : la carga oculta de salud. Mujer, salud y desarrollo. Washington, D.C.: O rganización Panamericana de la Salud, 1994:3-19.

9. De las zonas económicas catastrales para C iudadVictoria, D urango, D go Periódico 0 ficial del Gobierno Constitucional del Estado de Durango 1991 enero 11;11:83-88.

10. Ramírez RJC , Patiño G. Mujeres de G uadalajara y violencia doméstica: resultados de un estudio piloto. Cad Saude Publ 1996;12(3):405-409.

11. Kleinbaum DG, Kupper L, Morgenstern H. Epidemiology research. N uevaYork:Van N ostrand Reinhold, 1982:476-489.

12. Magnusson D.Teoría de los tests. México, D.F:Trillas. 1993:153-169.

13. Ferreira G B. La mujer maltratada. BuenosA ires: Editorial Sudamericana, 1988:25-41.

14.Valdez-Santiago R,Sanín-A guirre LH . La violencia do méstica durante el embarazo y su relación con el peso al nacer. Salud Publica Mex 1996;38:352-362. 